\title{
Worksheet-Driven UMM Modeling of B2B Services
}

\author{
Christian Huemer, Marco Zapletal \\ Institute of Software Technology \\ Vienna University of Technology \\ Favoritenstr. 9-11/188, 1040 Vienna, Austria \\ huemer@big.tuwien.ac.at,marco@ec.tuwien.ac.at
}

\author{
Philipp Liegl, Rainer Schuster \\ Austrian Research Centers GmbH - ARC \\ Research Studios Austria \\ Thurngasse 8/20, 1090 Vienna, Austria \\ \{pliegl, rschuster\}@ researchstudio.at
}

\begin{abstract}
In the development process of a B2B system it is crucial that the business experts are able to express and evaluate agreements and commitments between the partners and that the software engineers get all necessary information to bind the private process interfaces to the public ones. UN/CEFACT's modeling methodology (UMM) is a UML profile for developing $B 2 B$ processes. The formalisms introduced by UMM's stereotypes facilitate the communication with the software engineers. However, business experts - who usually have a very limited understanding of UML - prefer expressing their thoughts and evaluating the results by plain text descriptions. In this paper we describe an approach that presents an equivalent of the UMM stereotypes and tagged values in text-based templates called worksheets. This strong alignment allows an integration into a UMM modeling tool and ensures consistency. We show how a specially designed XML-based worksheet definition language allows customization to special needs of certain business domains. Furthermore, we demonstrate how information kept in worksheets may be used for the semi-automatic generation of pattern-based UMM artifacts.
\end{abstract}

\section{Motivation}

Within the recent past enterprises started to use service engineering concepts to build up a services portfolio representing their business. In this context services are economic activities offered to other business partners in order to achieve a certain benefit [30]. In most cases these services are intangible by nature and generated by business processes. Realizing the services portfolio in a technical sense results in $\mathrm{B} 2 \mathrm{~B}$ information systems according to the concept of a service-oriented architecture (SOA).

A successful B2B integration does not start with manually creating Web Services artifacts, such as WSDL or BPEL code. Such an approach does not consider the busi- ness perspective, the justification for the business process and the business requirements capturing the commitments and agreements between business partners. In addition, if business partners design their own interfaces in isolation, it is rather unlikely that their interfaces are complimentary to each other.

Instead, an approach is needed capturing the business process from a global perspective. It is necessary to focus on the business perspective of a $\mathrm{B} 2 \mathrm{~B}$ process by gathering business domain knowledge and business requirements. These requirements must be transformed into a flow of service interactions between business partners. Such an approach is envisioned by the UN/CEFACT Modeling Methodology (UMM). We have been a long-term contributor to UN/CEFACT and served as editing team for UMM's UML profile.

UN/CEFACT's Modeling Methodology (UMM) is an integrated approach for capturing the collaborative space between enterprises. The UMM was developed according to the Open-edi reference model [8]. It provides a modeling language and a methodology for accomplishing B2B projects. The methodology guides the analyst on his path from getting domain knowledge and requirements to designing business collaborations executed between business service interfaces. UMM's modeling language is defined as a profile on top of the Unified Modeling Language (UML) [20]. A UML profile specifies a set of stereotypes, tagged values and constraints for customizing UML. This means the general-purpose language UML is customized for the specific purpose of inter-organizational systems.

An advantage of UMM is the fact that it puts UML in a very strict corset. The resulting artifacts are well defined. Each artifact is restricted to a number of precisely defined modeling elements (stereotypes) and the relationships among them is also fixed. As a consequence, it is easier for software engineers to act upon the resulting artifacts in order to bind their local systems to the public process defined by UMM. However, it is rather hard for business experts to participate in the development of a UMM model. They usu- 
ally do not have any UML knowledge and are not able to produce artifacts according to the UMM profile. Communication with business experts is often based on plain text descriptions and less formal drawings. Today, a business analyst already uses some predefined templates - called worksheets - in order to gather information from the business experts. However, these worksheets are loosely connected to the UMM profile. Accordingly, the business analyst has to connect the dots - by combining information spread all over the worksheets - in order to create a UMM compliant model. In this paper we suggest a better alignment of worksheets and the UMM meta model. In fact, the worksheets must represent an equivalent of the UMM stereotypes and tagged values. We elaborate on the advantages of this approach and demonstrate it by means of a practical example.

The remainder of the paper is structured as follows: In section 2 we focus on the contributions of our approach. Firstly, it guarantees a single repository for worksheets and modeling artifacts. Secondly, it is flexible enough to cope with special needs of certain business domains. Thirdly, it enables the semi-automatic generation of UMM artifacts. Finally, it provides a consistent documentation of UMM models. In section 3 we demonstrate these advantages. We use the example of a waste management transport in order to go step by step through a UMM model. A short survey of related work is given in section 4 . Section 5 concludes the paper with a summary.

\section{Extending UMM by Worksheets}

\subsection{Aligning UMM stereotypes and work- sheets}

Worksheets provide effective means to persist existing business domain knowledge and business process knowledge. UN/CEFACT developed its current set of worksheets according to typical questions asked by a business analyst. As a result these worksheets are not perfectly aligned to the UMM meta model. In our approach we focus on a better alignment with UMM's UML profile. A UML profile specifies stereotypes, their tagged values, and OCL constraints on the UML meta model describing the relationships between the stereotypes. It is important that all stereotypes and tagged values are reflected in the worksheets - but not each stereotype has its own worksheet. This means that some stereotypes and their tagged values are described in the worksheet assigned to another stereotype - the master stereotype.

Since we align the worksheets and the UMM, we have a positive effect on the modeling environment. Today the worksheets are often completed on paper or by the means of a word processor. It is obvious that maintaining the textbased worksheets independent of the information stored in a UMM model results in a duplication of efforts and a high danger of inconsistencies. Since our worksheets are another way of presenting the UMM profile, we are able to integrate the worksheets into the UMM model. For these reasons we have developed a worksheet editor as a feature of our UMM Add-In [14]. Figure 1 shows an example of a worksheet in this editor. The editor supports the business analyst in capturing consistent business domain knowledge in an interactive manner. The worksheet editor stores the information directly in the tagged values of the corresponding stereotypes. In other words, we have built a single repository for the B2B system under consideration by combining UMM modeling artifacts and business requirements captured in worksheets.

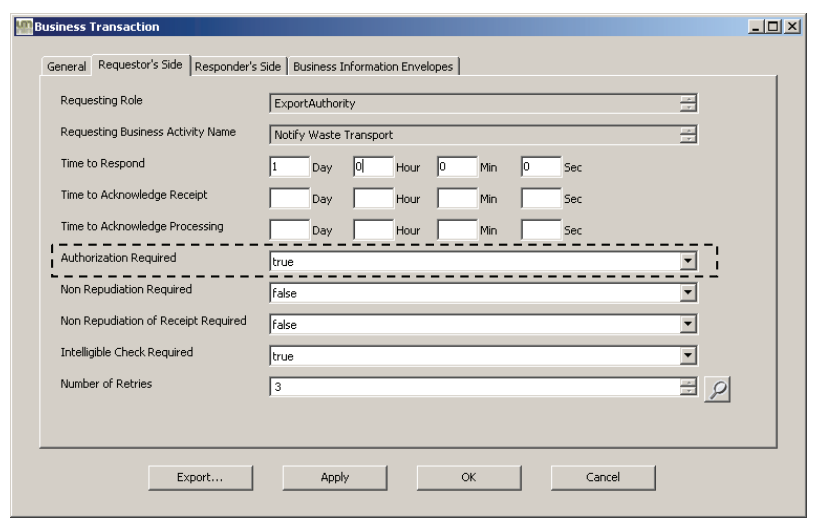

Figure 1. Worksheet editor: business transaction

\subsection{Customizing content and layout of worksheets}

Although we provide a pre-defined set of worksheets, it is not mandatory to use them. Different business domains usually have different business requirements to capture. The layout, the structure and the style of a worksheet is not hardcoded into our tool, instead these features are specified by the Worksheet Definition Language (WDL). Each UMM worksheet is based on a worksheet definition file specifying the nomenclature of the requirements entries, the clustering of entries into named categories, the design of the input boxes, the corresponding stereotypes, and their tagged values. In our tool we have created worksheet definition files in WDL format for our default worksheets matching the UMM profile. However, an business analyst may create new worksheets as well as extend or restrict existing worksheets according to special business needs.

The code below is an extraction of the worksheet definition file of a business transaction. It shows the tags related to the entry called authorization required. This entry 
is high-lighted by a dotted line in figure 1 . The entry is of type choice with the boolean values defined as elements between the choicebox tags. The tagged value name attribute specifies that the entry is stored in the tagged value of is authorization required. This tagged value is not part of the master stereotype business transaction, but defined within the related stereotype requesting business activity. Consequently, this stereotype is referenced in the attribute tagged value type. The name element defines the heading of the entry on the screen.

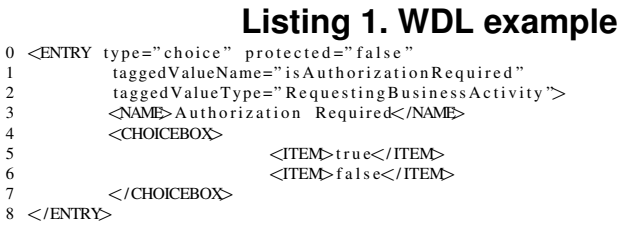

Our worksheet based approach also allows a very simple customization of the UMM meta model. As mentioned in the previous subsection, each worksheet entry is stored in a tagged value of a stereotype. WDL allows to specify the stereotype and the tagged value. Thus, a customization of a worksheet may include an entry which is not backed up by a tagged value of the standardized UMM meta model. Accordingly, the worksheet specification file has to specify a new tagged value of an existing or a new stereotype. Our tool dynamically loads the worksheet definition file, and if a tagged value is not part of the UMM meta model, it will create the tagged value on the fly. This approach allows for an easy update/customization of the meta model not requiring any changes of its profiles or code.

\subsection{Creating modeling artifacts from worksheet information}

The integration of the worksheets into a UMM modeling tool allows for another approach to produce UMM artifacts. The creation of UMM artifacts usually requires know-how in UML modeling and the specifics of the UMM profile. The artifacts are created on the modeling canvas of a modeling tool. In our approach the worksheets may drive the creation of some modeling artifacts. Since worksheets keep all the information about stereotypes and their tagged values, it is possible to (semi-)automatically create those artifacts that follow a pre-defined pattern. Following this approach, no special know-how in UML modeling is needed to create the artifacts. This feature relieves the business analyst from the burden of manually modeling routine tasks. We demonstrate this feature in section 3 by the example of a business entity state lifecycle and by the example of a business transaction.

\subsection{Documenting the Collaborative space of B2B models}

A good documentation is crucial in any software development project. A report on the UMM model should serve the needs of all stakeholders - from the business experts to the software engineers - with all their different skills and needs. Thus, it is not appropriate to limit a report only to the modeling artifacts. By having integrated the worksheets into the UMM modeling tool we are able to generate a documentation suitable for the evaluation by the business expert. The worksheet editor is able to generate an appropriate documentation in Microsoft Word, HTML or WDL based on the captured business domain knowledge. Using the WDL file format allows re-importing the data again into the worksheet editor for recovering purposes. In general, the documentation contains a textual, table-like description of the business knowledge. Different kinds of simple modeling artifacts may be added to these descriptions on demand.

\section{UMM and Worksheets by Example}

In this section we go step by step through the development process of a real world UMM model on managing waste transports in order to demonstrate our worksheetdriven approach. This model already takes advantage of UMM 2 [29] which eliminates some of the shortcomings of the current UMM standard [27]. According to UMM 2, a UMM model consists of three distinctive views: business requirements view, business choreography view and business information view. Each view comprises a set of packages and modeling artifacts. In this paper we are going to elaborate on the first two views, but do not go into detail of the business information view due to space limitations. Furthermore, it should be noted that - for reasons of readability - we do not present the screenshots of worksheets, but present equivalent table-like descriptions as produced by the export function.

\subsection{Business Requirements View (BRV)}

The business requirements view covers the business processes and their categorization into business areas and process areas. If necessary, a business process can be further detailed by using a business process activity model as depicted in figure 3. Before the business modeler starts to create the business process activity model the requirements of the process are captured in the business process worksheet as shown in figure 2. During the interview with the business domain expert the business analyst captures the necessary information of the business process and enters it into the worksheet. The information includes a definition and a description of the process as well as the participating parties 
and stakeholders. Furthermore pre- and post-conditions and start/end characteristics are stored in the worksheet. After the worksheet is filled out completely, the modeler starts to create the business process activity model according to the requirements elaborated before.

\begin{tabular}{|c|c|}
\hline \multicolumn{2}{|r|}{ Form: BusinessProcess } \\
\hline \\
\hline Business Process Name & Manage End-to-End Waste Transport \\
\hline Definition & $\begin{array}{l}\text { A waste transport taking place between an export authority and an import } \\
\text { authority. }\end{array}$ \\
\hline Description & $\begin{array}{l}\text { Subject of the business process is the waste transport between different } \\
\text { countries. The export authority of the export country pre-informs the import } \\
\text { authority of the import country about a waste transport. Upon successful } \\
\text { receipt of the waste transport the import authority informs the export } \\
\text { authority. }\end{array}$ \\
\hline Participants & ImportAuthority, ExportAuthority \\
\hline Stakeholder & none \\
\hline Reference & Waste Management \\
\hline \multicolumn{2}{|l|}{ Start/End Characteristics } \\
\hline Pre-condition & The waste is ready for transport. \\
\hline Post-condition & $\begin{array}{l}\text { - The waste has been moved from the export country to the import country. } \\
\text { - No waste transport took place. }\end{array}$ \\
\hline Begins When & Export authority receives the order to initiate the waste transport. \\
\hline Ends When & $\begin{array}{l}\text { The export authority receives the transport arrival receipt from the import } \\
\text { authority. }\end{array}$ \\
\hline Actions & $\begin{array}{l}\text { - Pre-inform on waste transport } \\
\text { - Inform on waste receipt }\end{array}$ \\
\hline Exceptions & - \\
\hline \multicolumn{2}{|l|}{ Relationships } \\
\hline Included Business Processes & none \\
\hline Affected Business Entities & WasteTransport \\
\hline
\end{tabular}

Figure 2. Business Process Worksheet

The activity diagram in figure 3 depicts the manage end-to-end waste transport. An exporter informs the export authority about a waste transport. The export authority in turn informs the import authority about the incoming waste transport. The import authority then informs the importer. Due to space limitations the activities of the exporter on the left hand side and of the importer on the right hand side are not shown in detail.

The export authority pre-informs the import authority about a waste transport. The import authority can either accept or reject the waste transport announcement. In case the waste transport announcement has been accepted the waste transport starts. On arrival of the waste in the import country the import authority informs the export authority about the arrived waste transport.

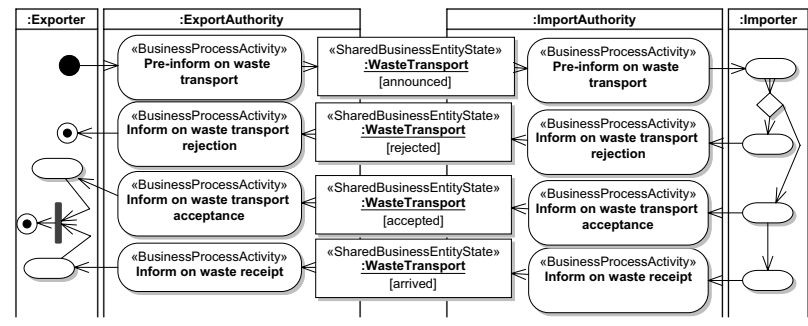

Figure 3. Business Process Activity Model

The information exchanged between the business partners in the business process is about the business entity waste transport. At first a business entity is created with state announced. The pending state announced is either set to approved or rejected by the responding import authority. After the approved transport happened the business entity is set to arrived. These so-called shared business entity states must be in accordance with the business entity lifecycle of waste transport as depicted in figure 5.

\begin{tabular}{|c|c|}
\hline \multicolumn{2}{|r|}{ Form: BusinessEntity } \\
\hline \multirow{2}{*}{\multicolumn{2}{|c|}{$\begin{array}{l}\text { General } \\
\text { Busines Fntity }\end{array}$}} \\
\hline Business Entity Name & \\
\hline Definition & $\begin{array}{l}\text { The waste transport business entity is the list of states a waste transport can } \\
\text { have. }\end{array}$ \\
\hline Description & A waste transport is taking place between an export and an import authority. \\
\hline \multicolumn{2}{|c|}{ BusinessEntityLifecycle } \\
\hline Pre-condition & A waste transport exists. \\
\hline Post-condition & The waste transport has been arrived or rejected. \\
\hline Begins When & A waste transport is initiated. \\
\hline Ends When & $\begin{array}{l}\text { The waste transport has successfully arrived or has been rejected by the } \\
\text { notifiee. }\end{array}$ \\
\hline Exceptions & - \\
\hline \multicolumn{2}{|l|}{ BusinessEntityState \#1 } \\
\hline Name & announced \\
\hline Definition & $\begin{array}{l}\text { A waste transport is in state "announced" if the notifiee has been informed } \\
\text { about it. }\end{array}$ \\
\hline Description & $\begin{array}{l}\text { Before the notifiee reports back to the notifier whether the waste transport is } \\
\text { accepted or rejected, the waste transport is in state "announced". }\end{array}$ \\
\hline Predecessing State & \\
\hline \multicolumn{2}{|l|}{ BusinessEntityState \#2 } \\
\hline Name & accepted \\
\hline Definition & $\begin{array}{l}\text { A waste transport is in state "accepted" if the notifiee positively responds to the } \\
\text { waste transport announcement of the notifier. }\end{array}$ \\
\hline Description & $\begin{array}{l}\text { Before the notifier is informed by the notifiee about the successful execution of } \\
\text { the waste transport, the waste transport is in state "accepted". }\end{array}$ \\
\hline Predecessing State & announced \\
\hline \multicolumn{2}{|l|}{ BusinessEntityState \#3 } \\
\hline Name & rejected \\
\hline Definition & $\begin{array}{l}\text { A waste transport is in state "rejected" if the notifiee negatively responds to the } \\
\text { waste transport announcement of the notifier. }\end{array}$ \\
\hline Description & $\begin{array}{l}\text { If the waste transport announcement is declined, the waste transport is in state } \\
\text { "rejected". }\end{array}$ \\
\hline Predecessing State & announced \\
\hline \multicolumn{2}{|l|}{ BusinessEntityState $\# 4$} \\
\hline Name & arrived \\
\hline Definition & $\begin{array}{l}\text { A waste transport is in state "arrived" if the waste transport was successfully } \\
\text { executed and the waste arrived in the target country. }\end{array}$ \\
\hline Description & $\begin{array}{l}\text { If the waste transport has been accepted by the notifiee and the transport was } \\
\text { executed successfully, the waste transport is in state "arrived". }\end{array}$ \\
\hline Predecessing State & accepted \\
\hline
\end{tabular}

Figure 4. Business Entity Worksheet

Both, the business entity life cycle and the business process activity model are strongly interlinked. Figure 4 depicts a worksheet capturing the requirements for a business entity lifecycle gathered during an interview between the business domain expert and the business analyst. First a definition and description of the business entity lifecycle is stored as well as pre- and post-conditions and begin/end characteristics. In the next step definitions and descriptions for every business entity state are gathered. The generation of the business entity life cycle as depicted in figure 5 is done automatically by the worksheet editor using the information of the business entity worksheet (figure 4). The entity graph is constructed by analyzing every life cycle state and its predecessing states. Furthermore the description and definition for every life cycle state as stored in the worksheet are transferred into tagged values of the model artifacts.

The worksheet for the business entity lifecycle is no arbitrary construct but designed according to the information stored in the UMM meta model. Figure 6 shows the business entity part of the UMM meta model. For our 


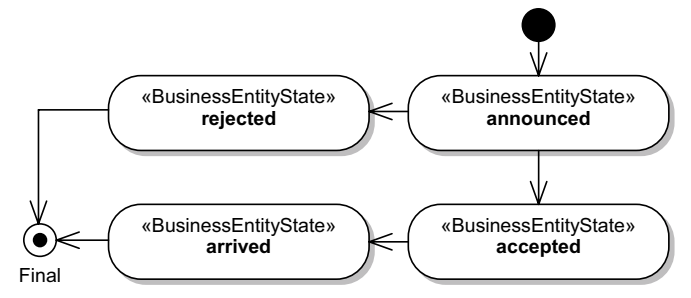

Figure 5. Business Entity Life Cycle

tool support we took the information from the meta model (attributes and associations) and transformed it into corresponding WDL constructs. These WDL file is then used to populate the correct worksheet.

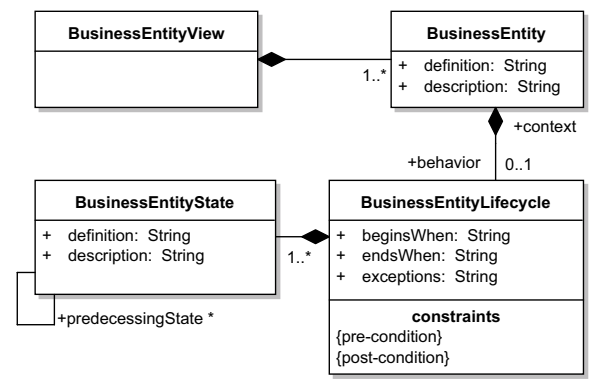

Figure 6. UMM Meta Model for Business Entities

\subsection{Business Choreography View (BCV)}

In the BRV the business analyst identified needs for communication in a collaborative business process by means of business process activity models and state changes of business entities. In the business choreography view the business analyst builds upon these artifacts in order to develop formal models of business collaboration protocols. In UMM, a business collaboration protocol is a choreography of business transactions.

The concept of a business transaction specifies the information exchange between exactly two business partners. The need for an information exchange is identified by the means of the shared business entity states in the BRV. It is the task of a business transaction to align the business information systems of the collaborating business partners. In other words, the business transaction is responsible for keeping all relevant business entities in the same state in both information systems.

Synchronization of states is either required in an unidirectional or in a bi-directional way. In the former case, the initiator of the business transaction informs the respond- ing partner about an already irreversible state change the responder has to accept - e.g., the notification that the waste has arrived. It follows, that responding in such a scenario is neither required nor reasonable. In the latter case, the initiating partner sets a business entity to an interim state and the responding partner decides about its final state - consider a request for a waste transport that the responder might either accept or refuse.

In our waste management example, we have identified four shared business entity states in the manage end-to-end waste transport business process activity model - announced, accepted, rejected, and arrived. Announced is a communicated interim state from the export authority that requires either the acceptance or the rejection of the waste transport. Thus, accepted and rejected represent final states. Given the business intention, the interim state announced and the two final states accepted and rejected result in one business transaction. We may call this business transaction announce waste transport. According to our business process activity model, the state arrived is communicated by the import authority to notify the export authority that the waste transport has arrived. This is done in the business transaction called announce transport arrival which represents a one-way message exchange. As shown in figure 7 the manage waste transport business collaboration consists of two distinctive business transactions namely announce waste transport and announce transport arrival.

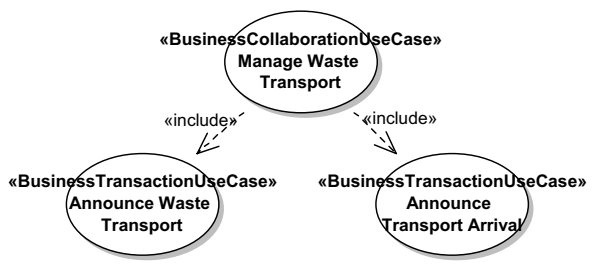

\section{Figure 7. Business Collaboration Require- ments}

In the remainder of this section, we will explain the semiautomatic generation of a business transaction using our worksheet editor by means of the example business transaction announce waste transport. Furthermore, we will outline how service specifications may be derived from fully elaborated business transaction models.

Business Transactions Developing a business transaction starts with defining a business transaction use case. The business transaction use case captures the formal requirements of the to-be-developed business transaction. Again, the business analyst applies worksheets to gather 
the know-how that is required to specify the aspects of a business transaction. According to our example, we define a business transaction use case announce waste transport. Its corresponding worksheet is given in figure 8. Beside the common information (name, definition and purpose) the worksheet gathers information such as the affected business entities - in our case waste transport - as well as the participating roles (export authority and import authority) and their activities (request waste transport and process waste movement form).

Based on the business transaction use case worksheet the worksheet editor creates the basic pattern of the business transaction. A business transaction is based on a UML activity diagram and follows always the same pattern: Two UML partitions are used to represent the two business partners. Each of them performs exactly one business action that sends and/or receives business information. The initiator's activity outputs information that is input to the responder's activity. The information flow in the reverse direction is optional.

\begin{tabular}{|l|l|}
\hline \multicolumn{2}{|l|}{ Form: BusinessTransactionUseCase } \\
\hline General & Announce Waste Transport \\
\hline Business Transaction Name & $\begin{array}{l}\text { "Announce Waste Transport" is used to provide the details of an intended } \\
\text { waste transport. }\end{array}$ \\
\hline Definition & $\begin{array}{l}\text { "Announce Waste Transport" deals with prior information } \\
\text { regarding the actual start of a waste shipment. The export authority } \\
\text { shall send signed copies of the completed movement document to } \\
\text { the competent authorities concerned at least three working days } \\
\text { before the shipment starts. The import authority either accepts the } \\
\text { notification or rejects it. }\end{array}$ \\
\hline Description & Export Authority \\
\hline Requesting Role & Import Authority \\
\hline Responding Role & Notify Waste Transport \\
\hline Requesting Activity & Process Waste Movement Form \\
\hline Responding Activity & Waste Movement \\
\hline Start/End Characteristics & $\begin{array}{l}\text { A general notification of the intended waste transport already } \\
\text { exists. }\end{array}$ \\
\hline Affected Business Entities & $\begin{array}{l}\text { Both the export authority and the import authority are aware of the } \\
\text { intended waste transport. }\end{array}$ \\
\hline Pre-condition & $\begin{array}{l}\text { The export authority receives a movement announcement request } \\
\text { and hence has to notify the import authority. }\end{array}$ \\
\hline Post-condition & \multicolumn{2}{|l}{} \\
\hline Begins When & $\begin{array}{l}\text { The export authority receives an acceptance or rejection from the } \\
\text { import authority. }\end{array}$ \\
\hline Actions & The time frame of the general notification is already exceeded. \\
\hline Ends When &
\end{tabular}

\section{Figure 8. Business Transaction Use Case Worksheet}

In case of announce waste transport we collected the information shown in figure 8 using the appropriate worksheet. Announce waste transport is performed between the export authority - playing the requesting role - and an import authority - taking up the responding role. The export authority performs the requesting business activity request waste transport and the import authority executes the responding business activity named process waste movement form. We already know that the execution of announce waste transport implies setting the business entity waste transport to an interim state (announced) before it is set to its final state (accepted or rejected). Thus, it contains a bi-directional flow of business information. Based on this information the worksheet editor generates the two partitions, assigns import authority and export authority as responsible for the respective partition and creates the requesting business activity request waste transport in the partition of the export authority and the responding business activity process waste movement form in the import authority's partition. This basic stub for a business transaction was fully created out of the corresponding business transaction use case worksheet (cf. figure 10. In order to complete the announce waste transport business transaction we require the following types of information: business transaction pattern, exchanged business information and quality of service parameters.

\begin{tabular}{|c|c|}
\hline \multicolumn{2}{|c|}{$\begin{array}{l}\text { Form: BusinessTransaction } \\
\end{array}$} \\
\hline \multicolumn{2}{|r|}{ 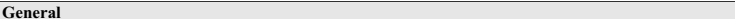 } \\
\hline Business Transaction Name & Announce Waste Transport \\
\hline Definition & $\begin{array}{l}\text { "Announce Waste Transport" is used to provide the details of an } \\
\text { intended waste transport. }\end{array}$ \\
\hline Description & $\begin{array}{l}\text { "Announce Waste Transport" deals with prior information } \\
\text { regarding the actual start of a waste shipment. The export } \\
\text { authority shall send signed copies of the completed } \\
\text { movement document to the competent authorities } \\
\text { concerned at least three working days before the shipment } \\
\text { starts. The import authority either accepts the notification or } \\
\text { rejects it. }\end{array}$ \\
\hline Select Business Transaction Pattern & Request/Confirm \\
\hline Secure Transport & true \\
\hline \multicolumn{2}{|l|}{ Requestor's Side } \\
\hline Requesting Role & Export Authority \\
\hline Requesting Business Activity Name & Notify Waste Transport \\
\hline \multirow{2}{*}{$\begin{array}{l}\text { Time to Respond } \\
\text { Time to Acknowledge Receipt }\end{array}$} & PTIDOH0M0S \\
\hline & null \\
\hline Time to Acknowledge Processing & null \\
\hline Authorization Required & true \\
\hline Non Repudiation Required & false \\
\hline Non Repudiation of Receipt Required & false \\
\hline Intelligible Check Required & true \\
\hline Number of Retries & 3 \\
\hline \multicolumn{2}{|l|}{ Responder's Side } \\
\hline Responding Role & Import Authority \\
\hline Responding Business Activity Name & Process Waste Movement Form \\
\hline Time to Acknowledge Receipt & null \\
\hline Time to Acknowledge Processing & null \\
\hline Authorization Required & true \\
\hline Non Repudiation Required & false \\
\hline Non Repudiation of Receipt Required & false \\
\hline \multirow{2}{*}{\multicolumn{2}{|c|}{\begin{tabular}{|l|l|} 
Intelligible Check Required & Business Information Envelopes \\
Bus
\end{tabular}}} \\
\hline & \\
\hline \multicolumn{2}{|c|}{ Information Envelope from Requesting Business Activity } \\
\hline \multicolumn{2}{|c|}{\begin{tabular}{l|l|l} 
& Information Name & WasteMovementFormEnvelope \\
\end{tabular}} \\
\hline Information State & \\
\hline Are Contents Confidential? & True \\
\hline Is the Envelope Tamperproof? & False \\
\hline \begin{tabular}{l|l|} 
& Authentication Required? \\
\end{tabular} & False \\
\hline Information Envelope from Respond & g Business Activity \\
\hline \begin{tabular}{l|l} 
Information Name \\
\end{tabular} & $\begin{array}{l}\text { WasteMovementAcceptanceEnvelope, } \\
\text { WasteMovementRejectionEnvelope }\end{array}$ \\
\hline Information State & \\
\hline Are Contents Confidential? & True \\
\hline Is the Envelope Tamperproof? & False \\
\hline Authentication Required? & False \\
\hline
\end{tabular}

\section{Figure 9. Business Transaction Worksheet}

The business transaction pattern defines the type of a legally binding interaction between two decision making applications as defined in Open-edi [8]. We distinguish between two one-way (information distribution, notification) and four two-way (query/response, request/response, request/confirm, commercial transaction) types of business transactions. The patterns differ in the default values of the tagged values characterizing a requesting/responding busi- 


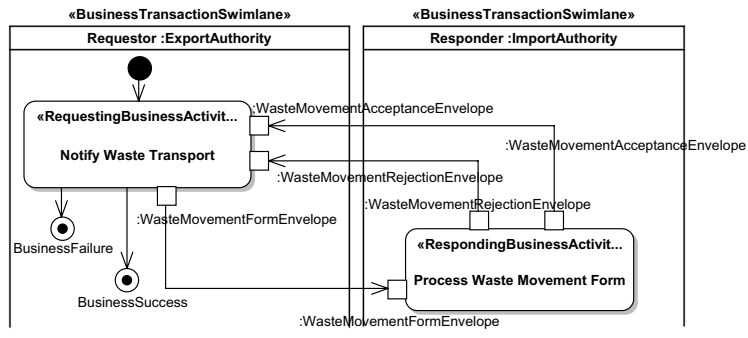

Figure 10. Waste Management Example: Business Transaction Announce Waste Transport

ness activity: is authorization required, is non-repudiation required, time to perform, time to acknowledge receipt, time to acknowledge acceptance, is non-repudiation of receipt required and retry count. These tagged values are considered as self-explanatory. Due to space limitations we refer the interested reader to the specification [27] for more information concerning these parameters.

Considering our example business transaction announce waste transport, the worksheet in figure 9 defines the respective quality of service parameters and the business transaction pattern. It is a request/confirm business transaction, because the export authority requests the waste transport, which is either confirmed or refused by the import authority playing the reacting part. The pattern of the business transaction dictates whether it is a one-way or a two-way message exchange. In case of a one-way message exchange, the worksheet editor permits only to define the request message. Otherwise, in case of a request-response business transaction the user is required to specify the type of the requesting business document as well as the responding business document types. Depending on the business intention of the business transaction, different response document types might be chosen to reflect a positive or a negative response to a request.

In order to define the exchanged business document types, the worksheet editor asks the business analyst to choose from a set of business document types. Modeling of business documents is part of the business information view in the UMM. Due to space limitations we do not elaborate the modeling of business information in this paper, but assume that business information is already present in our model. Considering our example worksheet in figure 9, the requesting business document sent by the export authority is of type waste transport movement form envelope. The import authority may either accept or reject the waste transport. Thus, we use a waste transport acceptance envelope to repre- sent a positive response. A rejection of the transport is signaled by a waste transport rejection envelope.

By having filled out the business transaction pattern, the exchanged business document types, and the quality of service parameters (or accepting their default values) we are ready to generate the entire business transaction from the worksheet information. In case of our example, the worksheet as given in figure 9 results in the business transaction shown in figure 10 .

In a nutshell, the worksheet approach together with the worksheet editor of our tool supports the user in modeling business transactions efficiently. The benefit of our approach is two-fold: Firstly, the business analyst is guided through the various steps needed to particularize the exchange of business information between business partners. This guidance prevents the business analyst from producing non-compliant artifacts. Secondly, the worksheet editor reliefs the modeler from recurrent modeling tasks. User input is elicited in a wizard-like manner and the resulting business transaction model is generated.

Business Collaborations A business transaction defines only the exchange of one message and its optional reply between business partners in a B2B process. We already learned that UMM uses the concept of a business collaboration protocol to represent complex collaborative processes. According to our waste management example, we have another business transaction in place dealing with the notification of the waste transport arrival - we may further call it announce transport arrival. The resulting business collaboration protocol - we call it manage waste transport - is a sequence of our example transaction announce waste transport and announce transport arrival. Due to space limitations we do not detail the announce transport arrival and the rather simple business collaboration protocol manage waste transport. For more information, the we refer the interested reader to our work in [5] and to the UMM specification [27].

Deriving software artifacts from UMM So far, we detailed the value of UMM for eliciting the business requirements in a B2B project. The tool-supported worksheet approach generates modeling artifacts from worksheet input to speed up the development of B2B processes. In this sub section, we show briefly how a software engineer benefits from UMM as stated at the beginning of this paper. Likewise generating modeling artifacts from worksheet input, a final UMM model provides the foundation for deriving software artifacts to support the implementation of a partner's abstract process. Consider again our example business transaction announce waste transport: If both partners - export authority and 
import authority-communicate through Web Services each party has to provide the respective services in order to fulfill its part of the public process. The service interface of the import authority has to expose an operation to receive the waste movement form envelope. Similarly, the export authority has to provide a service for the receipt of a waste movement acceptance envelope and a waste movement rejection envelope. Listings 2 and 3 shows stubs of WSDL port types for the service interfaces of the import authority and the export authority, respectively.

\section{Listing 2. WSDL port type for the import au- thority}

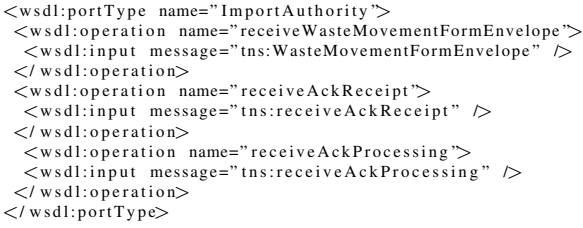

\section{Listing 3. WSDL port type for the export au- thority}

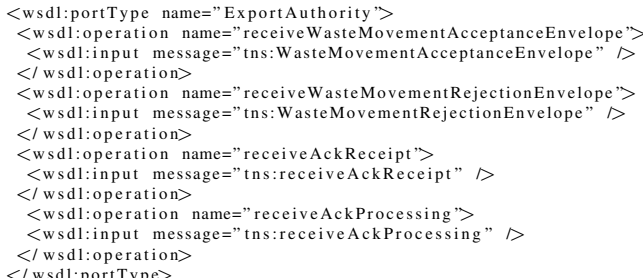

Beside the operation for receiving business documents both service interfaces define operations for receiving business acknowledgements. In UMM, business acknowledgements are defined using the quality of service parameters time to acknowledge receipt and time to acknowledge acceptance (cf. figure 9. The former one is sent, when a received business document passes sequence, grammar and schema validation. The latter one is sent when the business document passes checks against additional business rules. This example introduces only briefly the potential of a UMM model to derive artifacts for a software engineer. In [4] and [7] we proposed a mapping of UMM to ebXML BPSS and in [3] and [6] we outlined mappings to BPEL.

\section{Related work}

Over the last couple of years, a lot of methodologies for modeling business processes have been developed. Surveys comparing different types of business process modeling languages are provided in [15] [11]. Some of these approaches are based on special notations [25] [17] [21] [28]. Others customize the UML for business process modeling needs [16]. Most of these UML approaches are based on activity diagrams [24]. They either provide just guidelines on using activity diagrams for this special purpose or they specify a UML profile. Traditionally, business process modeling focuses on modeling business processes internal to an organization fulfilling customer needs [22]. More recent approaches also take inter-organizational business processes into account[10] [12]. Due to the growing importance of XML and Web Services, a lot of XML-based notations describing the orchestration and choreography of executable business processes have been developed. The most popular languages in this area are the Business Process Execution Language for Web Services (BPEL4WS) [19] [13] and the Business Process Specification Schema (BPSS) [26].

The advantage of a business based requirements approach has also been outlined in [18] and [1]. McGovern suggests the use of a business based approach in order to avoid a late design breakage and integration problems. A method similar to the requirements engineering approach with UMM is proposed in [2] using use case maps (UCMs). Such a scenario method allows the integration of different viewpoints e.g. business value viewpoint or business process viewpoint. Although providing an iterative process and a profound requirements engineering approach UCMs lack the possibility to generate artifacts out of the captured information.

A Business Media Framework providing a solution for the integrated management of business transactions is presented in [23]. Next to the business requirements, the model presented also considers security and legal aspects in detail. A framework for service-oriented software engineering has been presented in [9]. The idea of the framework is to create a well defined business case in which the business processes and the business requirements are identified by using UML and BMPL diagrams. Similar to the approach with use case maps this framework lacks the semi-automatic generation of artifacts out of the collected information.

\section{Conclusion}

In this paper, we have shown an approach that improves requirements engineering as part of the UN/CEFACT's Modeling Methodology for specifying B2B systems. Our approach helps to avoid inconsistencies between worksheets capturing the business experts' knowledge and the modeling artifacts. This is realized by integrating a worksheet editor into our UMM modeling tool.

The worksheets are not hard-coded into the modeling tool. Instead XML-based worksheet definition files are dy- 
namically loaded into the modeling tool. We introduce the Worksheet Definition Language (WDL) that allows the creation of new worksheets and the customization of existing worksheets.

Another feature of our worksheet editor is the semiautomatic generation of modeling artifacts in order to speed up the modeling process and to ensure consistent results. Finally, special report generation functions allow a validation of the B2B system specification by the business experts. By the work outlined in this paper, we improved the overall quality of requirements engineering in UMM-based B2B projects.

\section{References}

[1] M. Erder and P. Pureur. Defining business requirements quickly and accurately. IT Professional, 6:51-56, 2004.

[2] J. Gordijn, H. de Bruin, and H. Akkermans. Scenario Methods for Viewpoint Integration in e-Business Requirements Engineering. In Proceedings of the 34th Hawaii International Conference on System Sciences. IEEE, 2001.

[3] B. Hofreiter and C. Huemer. Transforming UMM Business Collaboration Models to BPEL. In Proceedings of OTM Workshops 2004. Springer LNCS, 2004.

[4] B. Hofreiter, C. Huemer, and J.-H. Kim. Choreography of ebXML business collaborations. Information Systems and e-Business Management (ISeB), June 2006.

[5] B. Hofreiter, C. Huemer, P. Liegl, R. Schuster, and M. Zapletal. UN/CEFACT'S Modeling Methodology (UMM): A UML Profile for B2B e-Commerce. In Advances in Conceptual Modeling - Theory and Practice, ER 2006 Workshops BP-UML. Springer LNCS, 2006.

[6] B. Hofreiter, C. Huemer, P. Liegl, R. Schuster, and M. Zapletal. Deriving executable BPEL from UMM Business Transactions. In Proceedings of the IEEE International Conference on Services Computing (SCC 2007). IEEE, 2007.

[7] M. Ilger and M. Zapletal. An Implementation to transform Business Collaboration Models to executable Process Specifications. In Proceedings of the Conference on Service-Oriented Electronic Commerce at the Multikonferenz. Wirtschaftsinformatik 2006, Passau Germany, Lecture Notes in Informatics (LNI), pages 9-23, 2006.

[8] ISO. Open-edi Reference Model, 2004. ISO/IEC JTC 1/SC30 ISO Standard 14662, Second Edition.

[9] H. Karhunen, M. Jäntti, and A. Eerola. Service-Oriented Software Engineering (SOSE) Framework. In Proceedings of 2005 International Conference on Services Systems and Services Management (ICSSSM). IEEE, 2005.

[10] H. Kim. Conceptual Modeling and Specification Generation for B2B Business Processes based on ebXML. SIGMOD Rec., 31(1):137-145, 2002.

[11] B. Korherr and B. List. An Evaluation of Conceptual Business Process Modelling Languages,. In 21st ACM Symposium on Applied Computing (SAC'06). ACM Press, 2006.

[12] G. Kramler, E. Kapsammer, G. Kappel, and W. Retschitzegger. Towards Using UML 2 for Modelling Web Service Collaboration Protocols. In Proceedings of the First Interna- tional Conference on Interoperability of Enterprise Software and Applications (INTEROP-ESA'05), Feb. 2005.

[13] F. Leymann, D. Roller, and M.-T. Schmidt. Web services and business process management. IBM Systems Journal New Developments in Web Services and E-commerce, 41(2), 2002.

[14] P. Liegl, R. Schuster, and M. Zapletal. UMM AddIn. Research Studios Austria, 2007. Version 0.8.2, http://ummaddin.researchstudio.at.

[15] F.-R. Lin, M.-C. Yang, and Y.-H. Pai. A generic structure for business process modeling. Business Process Management Journal, 8(1):19-41, 2002.

[16] B. List and B. Korherr. A UML 2 Profile for Business Process Modelling. In ER 2005 Workshops Proceedings, 2005.

[17] R. Mayer, C. Menzel, M. Painter, P. deWitte, T. Blinn, and B. Perakath. Information Integration for Concurrent Engineering (IICE) IDEF3 Process Description Capture Method Report. Technical report, Sept. 1995.

[18] F. McGovern. Managing software projects with businessbased requirements. IT Professional, 4:18-23, 2002.

[19] OASIS Web Services Business Process Execution Language (WSBPEL) TC. Web Services Business Process Execution Language Version 2.0, Jan. 2007. Version 2.0, Commitee Specification.

[20] Object Management Group (OMG). Unified Modeling Language Specification, Apr. 2005. Version 1.4.2.

[21] Object Management Group (OMG). Business Process Modeling Notation, 2006. Specification, Version 1.0.

[22] M. Penker and H.-E. Eriksson. Business Modeling With UML: Business Patterns at Work. Wiley, 2000.

[23] A. Rungeand, B. Schopp, and K. Stanoevska-Slabeva. The Management of Business Transactions through Electronic Contracts. In Proceedings of the 10th Internaional Workshop on Database and Expert Systems Applications. IEEE, 1999.

[24] N. Russell, W. M. van der Aalst, A. H. ter Hofstede, and P. Wohed. On the Suitability of UML 2.0 Activity Diagrams for Business Process Modelling. In Third Asia-Pacific Conference on Conceptual Modelling (APCCM2006). Australian Computer Society, Inc., 2006.

[25] A.-W. Scheer. ARIS - Business Process Modeling. Springer, 2000.

[26] UN/CEFACT. UN/CEFACT - ebXML Business Process Specification Schema, Nov. 2003. Version 1.11.

[27] UN/CEFACT. UN/CEFACT's Modeling Methodology (UMM), UMM Meta Model - Foundation Module, Mar. 2006. Technical Specification V1.0, http://www.unece.org/cefact/umm/UMM_Foundation_Module.pdf.

[28] W. M. P. van der Aalst. Making work flow: On the application of petri nets to business process management. In $A p$ plications and Theory of Petri Nets 2002, 23rd International Conference, ICATPN 2002. Springer LNCS, 2002.

[29] M. Zapletal, R. Schuster, P. Liegl, C. Huemer, and B. Hofreiter. UMM - A UML Profile for B2B e-Commerce - Featuring new concepts for a move towards UML 2. submitted, http://www.ec.tuwien.ac.at/ $/$ marco/pub/UMMAUmlProfile4B2BeCommerce.pdf, 2007.

[30] V. Zeithaml, M. J. Bitner, and D. D. Gremler. Services Marketing. McGraw-Hill/Irwin, New York, NY, May 2005. 